\title{
Recent results on hadronic final states from Babar
}

\author{
J. William Gary ${ }^{1, a}$ \\ ${ }^{1}$ Department of Physics and Astronomy, University of California, Riverside CA, 92521, USA
}

\begin{abstract}
Two recent studies from the Babar Collaboration at SLAC are presented on the production of hadrons at low energies. The first is a study of exclusive $K^{+} K^{-}$production in $e^{+} e^{-}$annihilation events with initial-state photon radiation. The second is a study of $\eta_{c}$ production in two-photon interactions and a three-body Dalitz-plot analysis searching for intermediate scalar meson production in $\eta_{c}$ decays.
\end{abstract}

\section{Introduction}

The Babar experiment operated at the PEP-II asymmetricenergy $e^{+} e^{-}$collider at SLAC from 1999 to 2008. The data analysis is still very active, with around 20 papers expected to be submitted for publication in 2014. The bulk of the data sample was collected at the energy of the $\Upsilon(4 S)$ resonance, with smaller samples collected at the $\Upsilon(2 S)$, the $\Upsilon(3 S)$, and at an off-resonance energy slightly below the $\Upsilon(4 S)$. Here, two recent studies of hadronic final states are presented. The first [1] is a study of the low-energy exclusive $e^{+} e^{-} \rightarrow K^{+} K^{-}$cross section based on events with untagged initial-state photon radiation (ISR), leading to a precise measurement of the charged kaon electromagnetic form factor and a test of QCD. The second [2] is a study of the two-photon processes $e^{+} e^{-} \rightarrow e^{+} e^{-} K^{+} K^{-} \eta$ and $e^{+} e^{-} \rightarrow e^{+} e^{-} K^{+} K^{-} \pi^{0}$, leading to observations of the $\eta_{c}$ meson and its decay to scalar mesons.

\section{The $e^{+} e^{-} \rightarrow K^{+} K^{-}$cross section and charged kaon form factor}

Until recently, there have been essentially no measurements of the charged kaon form factor. In 2005 the CLEO Collaboration published a result [3] on the charged kaon form factor at the fixed energy $\sqrt{s}=3.7 \mathrm{GeV}$. This was followed in 2013 by results [4] from Seth et al. (using CLEO data) at the fixed energies of 3.8 and $4.2 \mathrm{GeV}$. In 2013, the Babar Collaboration published results [5] using the ISR method, in which a photon radiated from the initial $e^{+}$or $e^{-}$in the $e^{+} e^{-}$annihilation process reduces the energy available to the $K^{+} K^{-}$system, allowing measurement of the form factor over a range of energies $\sqrt{s^{\prime}}$. The results of these previous studies are summarized in Fig. 1. Figure 1 includes the leading-order asymptotic QCD prediction, which is seen to lie well below the data.

The Babar form factor results in Ref. [5] are based on tagged ISR photons, in which the photon is observed

\footnotetext{
a e-mail: bill.gary@ucr.edu
}

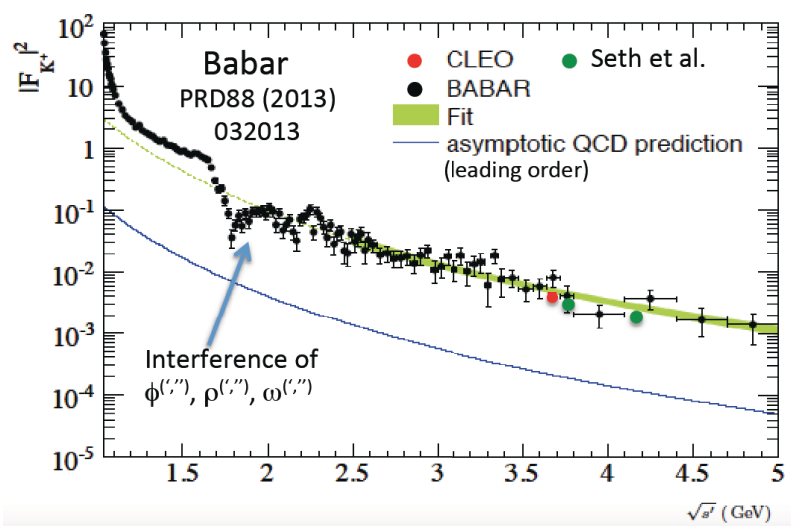

Figure 1. Previous measurements [3-5] of the charged kaon factor (squared), in comparison with the QCD asymptotic prediction.

within the fiducial volume of the detector. The new study, presented here, is based on untagged ISR photon events, i.e., on events with photons produced at angles close to the beam axis, outside the detector acceptance. The untagged analysis allows larger dikaon invariant mass values $m_{K K}=\sqrt{s^{\prime}}$ to be probed. The resulting data lie closer to the asymptotic regime and thus allow a more valid test of the QCD prediction.

The analysis is based on $469 \mathrm{fb}^{-1}$ of data collected at the $\Upsilon(4 S)$ resonance or slightly below. Events are required to contain exactly two charged tracks with opposite charges, each of which is identified as a kaon using a combination of information from a ring-imaging detector and the central tracking chamber. The two tracks are fit to a common vertex with a beam-spot constraint. The $\chi^{2}$ probability of the fit is required to exceed $0.1 \%$. Event are selected that are consistent with an ISR photon along the beam direction. Specifically the magnitude $P_{T, K K}$ of the vector sum of the transverse momenta of the two kaons is required to be less than $0.15 \mathrm{GeV}$, and the magnitude of the missing mass squared $M_{\text {miss }}^{2}=\left|\left(p_{e^{+}}+p_{e^{-}}-p_{K K}\right)^{2}\right|$ (with 

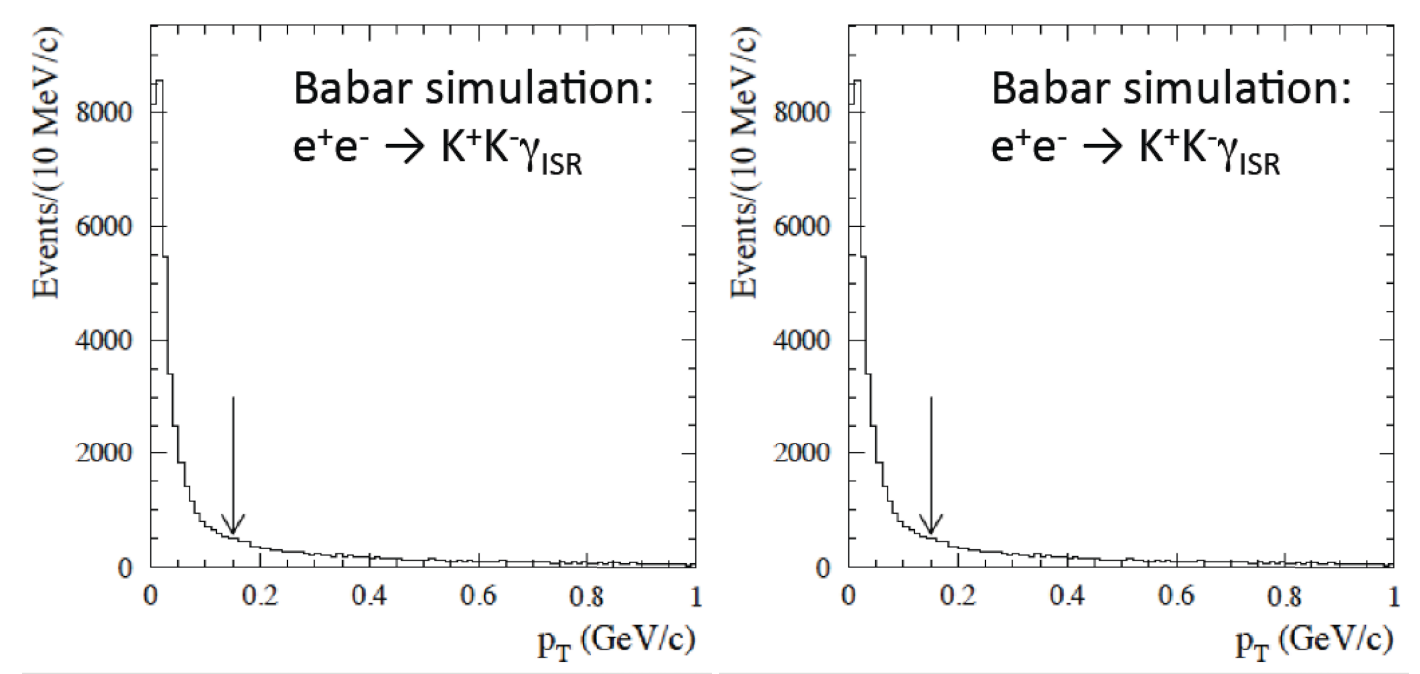

Figure 2. Distributions of (left) the magnitude $P_{T, K K}$ of the vector sum of the transverse momenta of the two kaons, and (right) the missing mass squared $M_{\text {miss }}^{2}$, for simulated $e^{+} e^{-} \rightarrow K^{+} K^{-} \gamma_{I S R}$ signal events. The arrows indicate selection requirements.

$p_{i}$ the 4-momenta) is required to be less than $1 \mathrm{GeV}$. The expected distributions of these two variables for simulated signal events are shown in Fig. 2.

The dikaon invariant mass distribution $M_{K K}$ of events selected with these requirements is shown in Fig. 3 (left). A clear $J / \psi$ peak is visible. For $M_{K K}>6 \mathrm{GeV}$, a large background is present from $e^{+} e^{-} \rightarrow \mu^{+} \mu^{-} \gamma_{I S R}$ events. Note that muon identification has not been applied at this stage. The green-shaded histogram in Fig. 3 (left) shows the distribution of events that have at least one identified muon. Events with at least one muon candidate are rejected. After applying this (final) selection requirement, the signal detection efficiency ranges from 1-14\%, depending on $M_{K K}$.

The analysis is restricted to values $M_{K K}<8 \mathrm{GeV}$ because the signal rate is very small for larger $M_{K K}$ values. Also, the $e^{+} e^{-} \rightarrow \mu^{+} \mu^{-} \gamma_{I S R}$ background becomes large as $M_{K K}$ approaches the nominal $e^{+} e^{-}$collision energy $\sqrt{s}$.

Background from $e^{+} e^{-} \rightarrow e^{+} e^{-} \gamma_{I S R}, \pi^{+} \pi^{-} \gamma_{I S R}$, and $p \bar{p} \gamma_{I S R}$ events is evaluated with control samples and found to be negligible. Other ISR background, e.g., from $e^{+} e^{-} \rightarrow$ $K^{+} K^{-} \pi^{0} \gamma_{I S R}$ events, is evaluated using data sideband distributions in the $M_{\text {miss }}^{2}$ and $p_{T, K K}$ variables. The background from untagged $e^{+} e^{-} \rightarrow e^{+} e^{-} K^{+} K^{-}$two-photon processes is obtained from simulation normalized to data.

The principal background in the signal region arises from residual $e^{+} e^{-} \rightarrow \mu^{+} \mu^{-} \gamma_{I S R}$ events. To evaluate this background, a maximum-likelihood fit is performed to the $M_{m i s s}^{2}$ distribution in bins of $M_{K K}$. The $M_{m i s s}^{2}$ distribution peaks at zero for signal events and at negative values for $e^{+} e^{-} \rightarrow \mu^{+} \mu^{-} \gamma_{I S R}$ events. The probability distribution function (PDF) for the $e^{+} e^{-} \rightarrow \mu^{+} \mu^{-} \gamma_{I S R}$ background component is obtained from $\mu^{+} \mu^{-} \gamma$ events with one identified muon. The PDF for the signal component is obtained from simulation.

The signal yield is extracted from the fit and translated into a measurement of the signal cross section
$\sigma_{K^{+} K^{-}}$as a function of $M_{K K}$ by dividing the signal yield by the efficiency and luminosity, calculated individually for each $M_{K K}$ bin. The signal cross section is then used to determine the magnitude of charged kaon form factor $\left|F_{K K}\right|$ using the relation $\sigma_{K K}\left(M_{K K}\right)=$ $\left[\pi \alpha^{2} \beta^{3} C /\left(3 M_{K K}^{2}\right)\right]\left|F_{K}\left(M_{K K}\right)\right|$, where $\beta=\sqrt{1-4 m_{K}^{2} / M_{K K}^{2}}$ and $C \approx 1.0008$ is a final-state Coulomb correction.

The results for $M_{K K}<5 \mathrm{GeV}$ are shown in comparison with the previous results in Fig. 3 (center). The untagged (SA ISR, or "small angle") results are seen to be much more precise that the tagged (LA ISR, or "large angle") results presented by Babar previously. The results for $M_{K K}>2.6 \mathrm{GeV}$ are shown in Fig. 3 (right). The untagged results extend the form factor measurements to much higher energies than the previous studies. Figure 3 (right) includes the QCD predictions: the leading-order asymptotic [Asy (LO)] result [6] mentioned above, the next-to-leading-order asymptotic [Asy (NLO)] result, and a next-to-leading-order [CZ (NLO)] calculation [7] that accounts for quark motion within the hadrons (finite quark masses). At the higher scales probed by this analysis, the QCD prediction is seen to be in much better agreement with the data than at the lower masses previously considered.

\section{$3 \eta_{c}$ production and scalar mesons}

The second study is based on an entirely different class of events, namely untagged two-photon events $e^{+} e^{-} \rightarrow$ $e^{+} e^{-} X$, which give access to $J^{P C}$ states not directly produced in $e^{+} e^{-}$annihilations. In particular, the $\eta_{c}$ meson, with $J^{P C}=0^{-+}$, can be produced directly in the twophoton process. The focus of the study presented here is scalar meson production in $\eta_{c}$ decays. The scalar meson sector has long been problematic, with more states observed than can be accommodated by the quark model, and with broad and interfering states that are difficult to inter- 

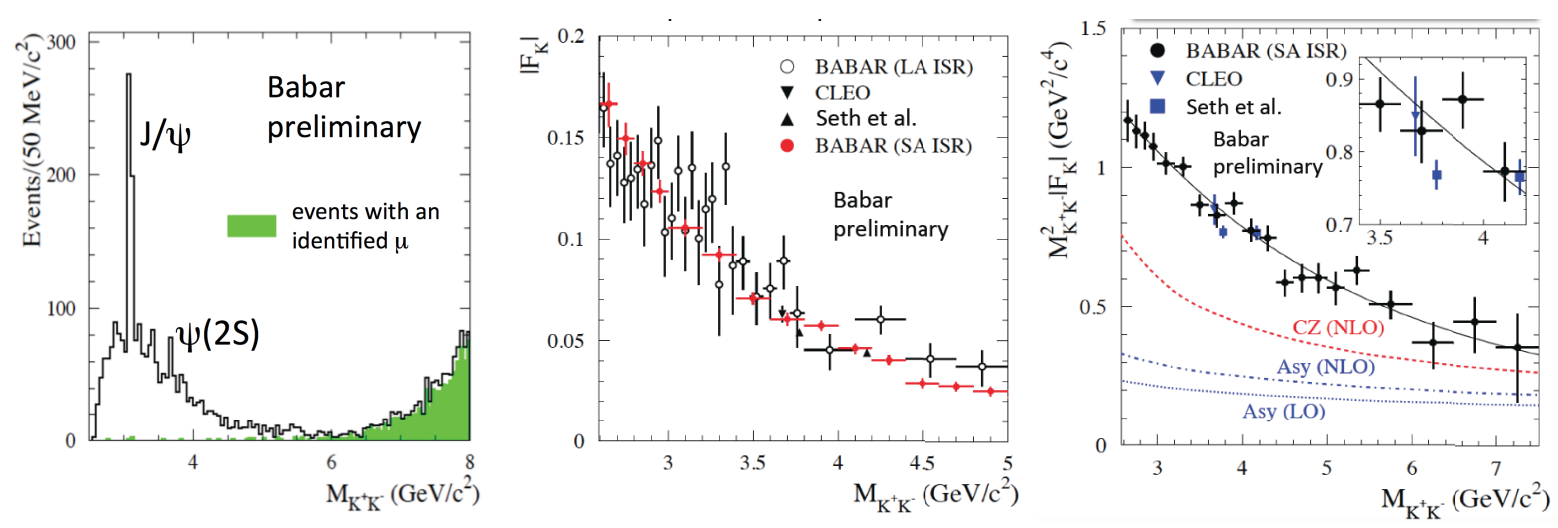

Figure 3. (left) Dikaon mass distribution $M_{K K}$ of selected events. (center) Charged kaon form factor results for $M_{K K}<5 \mathrm{GeV}$ in comparison with previous measurements. (right) Charged kaon form factor results for $M_{K K}>2.6 \mathrm{GeV}$ in comparison with QCD calculations.

pret. Searches for scalar glueballs have been performed in $J / \psi$ decays. This study allows a search for glueball candidates in $\eta_{c}$ decays.

The analysis is based on $519 \mathrm{fb}^{-1}$ of data, representing effectively the entire Babar data sample: the $\Upsilon(n S)$ $(\mathrm{n}=1,2,3)$ and off-peak samples. The targeted reactions are 1) $\gamma \gamma \rightarrow K^{+} K^{-} \eta$ with $\eta \rightarrow \gamma \gamma$, 2) $\gamma \gamma \rightarrow K^{+} K^{-} \eta$ with $\eta \rightarrow \pi^{+} \pi^{-} \pi^{0}$, and 3) $\gamma \gamma \rightarrow K^{+} K^{-} \pi^{0}$. For reactions 1) and 3 ), we require exactly two charged tracks and for reaction 2) exactly four charged tracks, with identification of the tracks as kaons or pions, as appropriate. The tracks are fit to a common vertex with a beam-spot constraint. The $\chi^{2}$ probability of the fit is required to exceed $0.1 \%$. The tracks are combined with a $\eta \rightarrow \gamma \gamma$ or $\pi^{0} \rightarrow \gamma \gamma$ candidate. The selected photon pairs must satisfy a beam-spot constraint and yield a diphoton mass consistent with the $\eta$ or $\pi^{0}$ mass. Analogous requirements are applied to reconstruct $\eta \rightarrow \pi^{+} \pi^{-} \pi^{0}$ candidates.

Events consistent with $\gamma \gamma \rightarrow K^{+} K^{-}$events are rejected by eliminating events with $p_{T, K K}<0.1 \mathrm{GeV}$. Background from the ISR processes $e^{+} e^{-} \rightarrow K^{+} K^{-} \eta \gamma_{I S R}$ and $K^{+} K^{-} \pi^{0} \gamma_{I S R}$ is reduced by requiring the missing-mass squared to exceed $10 \mathrm{GeV}$.

The transverse momentum of the candidate $K^{+} K^{-} \eta$ and $K^{+} K^{-} \pi^{0}$ systems are shown in Fig. 4. The results are shown in comparison with simulations of the targeted signal processes. Peaks at low transverse momentum are seen for all three reactions. The shapes of the peaks are consistent with the simulated signal shapes. To select events, we require the transverse momentum of the targeted threeparticle systems to be less than $0.05 \mathrm{GeV}$ (see the dashed vertical lines in Fig. 4). The signal selection efficiency is around $5 \%$ and is evaluated as a function of $m_{K K}$ and the $K^{+}$helicity angle.

The resulting $K^{+} K^{-} \eta$ and $K^{+} K^{-} \pi^{0}$ three-body invariant mass spectra are shown in Fig. 5. Very large signal peaks are seen for the $\eta_{C}$ meson. Clear $\eta_{c}(2 S)$ peaks are also seen. These results provide the first observation of $\eta_{c} \rightarrow K^{+} K^{-} \eta$ decays. The only previous result [8] for this channel is based on a signal yield of only $6.7 \pm 3.2$ events, and is not significant. We also obtain the first evidence for $\eta_{c}(2 S) \rightarrow K^{+} K^{-} \eta$ decays (4.9 standard deviations of significance, including systematics) and $\eta_{c}(2 S) \rightarrow K^{+} K^{-} \pi^{0}$ decays (3.7 standard deviations).

We perform a Dalitz-plot analysis of $\eta_{c} \rightarrow K^{+} K^{-} \eta$ decays, in order to search for resonant structure. Events in the $\eta_{c}$ mass range are selected for this study, yielding a sample with $1161 \eta_{c} \rightarrow K^{+} K^{-} \eta$ candidates and $76 \%$ purity. A maximum-likelihood fit is performed based on a sum of amplitudes, each representing a possible intermediate resonant state. The fit determines the relative magnitudes and phases of the amplitudes, with background contributions evaluated using sideband data in the $K^{+} K^{-} \eta$ mass distribution. The fit is performed assuming no interference between the signal and background components.

Figure 6 (left) shows the projection of the fit in the $m_{K K}^{2}$ variable. We observe clear evidence for scalar meson production in the intermediate states. Specifically, prominent mass peaks are seen for the $f_{0}(980), f_{0}(1500), f_{0}(1710)$, and $f_{0}(2200)$ mesons. The $f_{0}(1500)$ and $f_{0}(1710)$ are glueball candidates, and the strength of their coupling to the $\eta_{c}$ is important to help establish their character. The projection of the Dalitz-plot results onto the $m_{K \pi}^{2}$ variable is shown in Fig. 6 (right). In this case, peaks for the $K_{0}^{*}(1430)$ and $K_{0}^{*}(1950)$ are seen. These two resonances had previously been seen only in the $K \pi$ decay channel. The $K_{0}^{*}(1430)^{+} \rightarrow K^{+} \eta$ measurement, seen with a significance of 10.3 standard deviations, represents the first observation of this decay mode. Thus the $\eta_{c} \rightarrow K^{+} K^{-} \eta$ amplitude is dominated by scalar meson production.

We perform a similar Dalitz-plot analysis of $\eta_{c} \rightarrow$ $K^{+} K^{-} \pi^{0}$ decays. After selecting the region around the $\eta_{c}$ mass, we obtain 6710 events with $55 \%$ purity. The background is evaluated with sidebands in the three-particle invariant mass distribution, as with the $\eta_{c} \rightarrow K^{+} K^{-} \eta$ analysis. The projections of the results onto the $m_{K \pi^{0}}^{2}$ and $m_{K K}^{2}$ axes are shown in Fig. 7. Scalar amplitudes are seen to dominate for this process also, with strong $K_{0}^{*}(1430)$ and $K_{0}^{*}(1950)$ components in the $K \pi$ mode and $a_{0}$ production in the $K K$ mode [note that the vector $K^{*}(890)$ peak seen in Fig. 7 (left) is consistent with the expectation from background]. 

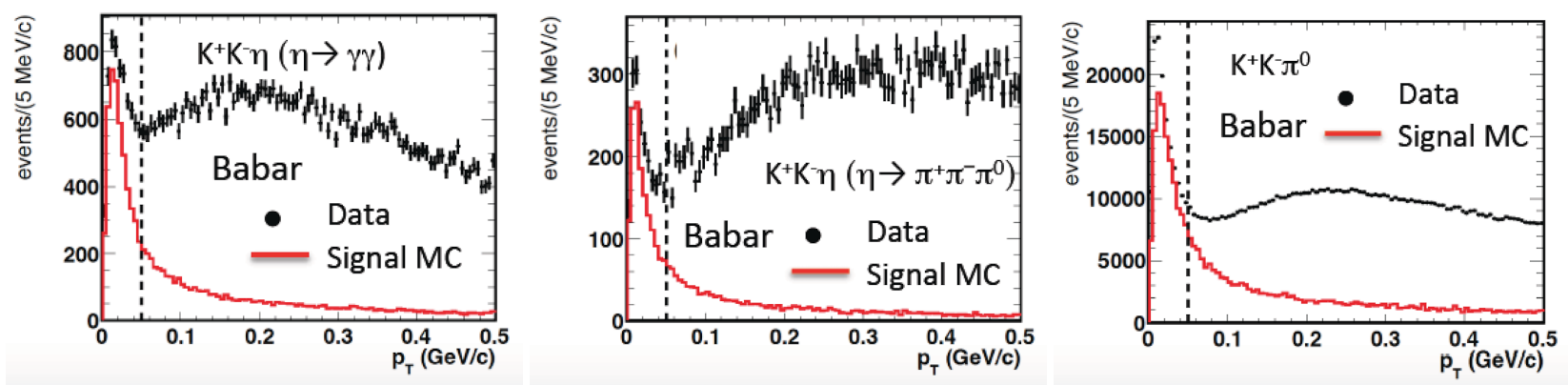

Figure 4. Transverse momentum distributions of the candidate $K^{+} K^{-} \eta$ and $K^{+} K^{-} \pi^{0}$ systems in comparison with the corresponding simulations of signal events. The dashed lines indicate the selection requirements.
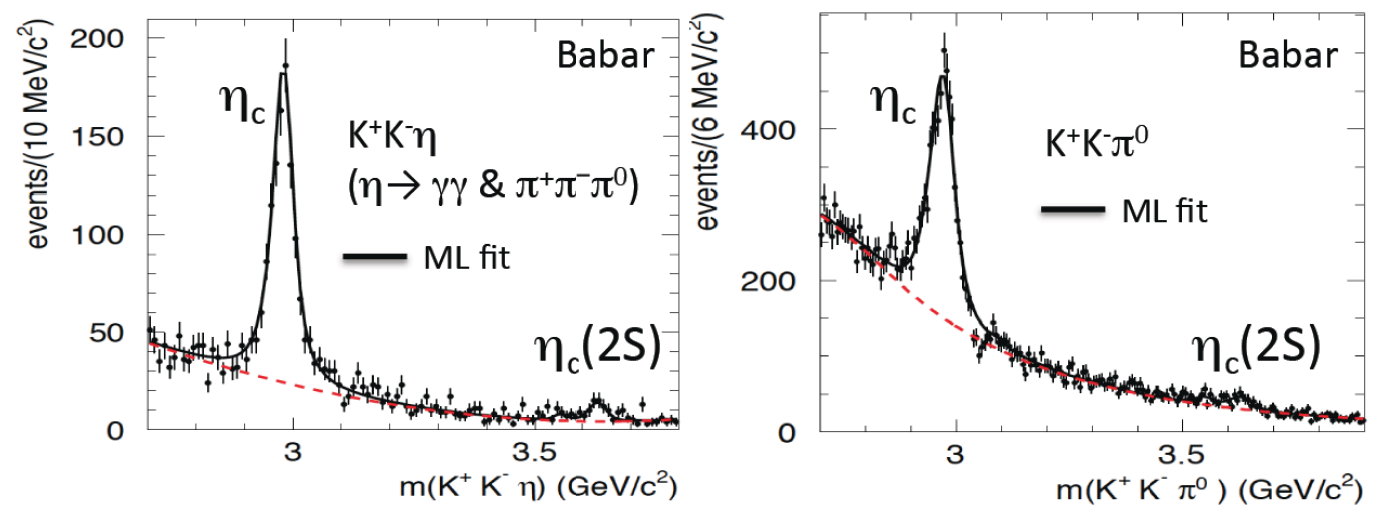

Figure 5. Invariant mass distributions of the selected (left) $K^{+} K^{-} \eta$ and (right) $K^{+} K^{-} \pi^{0}$ systems, along with the results of the maximumlikelihood fit.
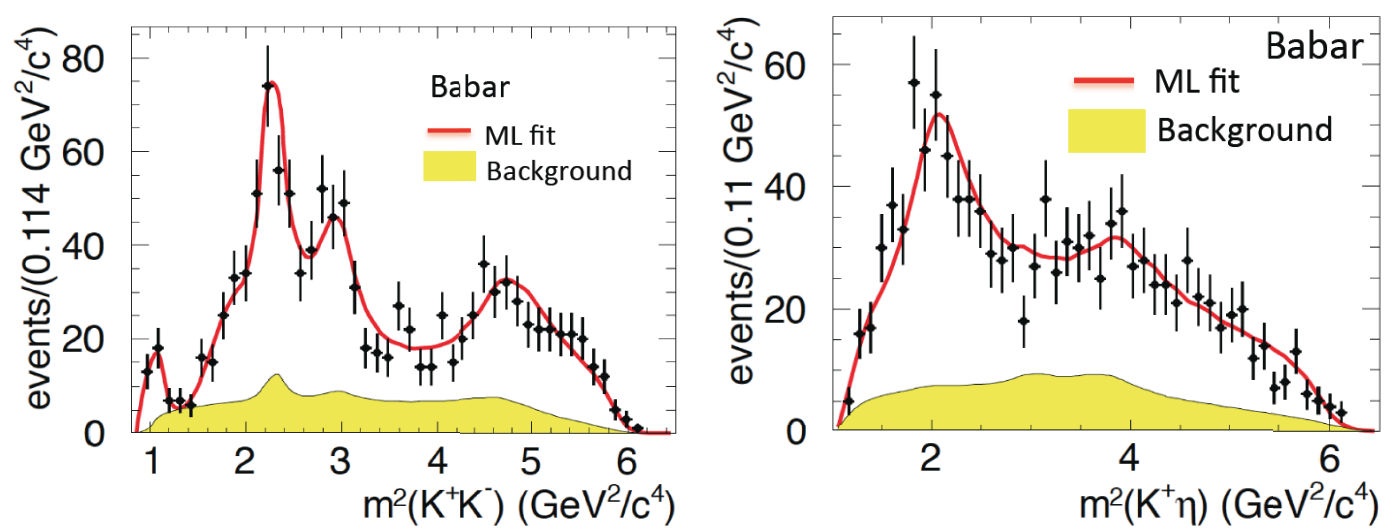

Figure 6. Projection of the Dalitz plot results for $\eta_{c} \rightarrow K^{+} K^{-} \eta$ events onto the (left) $m_{K K}^{2}$ and (right) $m_{K \pi}^{2}$ axes.

Finally, we present measurements of the $K_{0}^{*}(1430)$ mass and width. These measurements are obtained using scans. The motivation for these measurements is that there are large uncertainties in the PDG averages for the $K_{0}^{*}(1430)$ resonance parameters. The large PDG uncertainties arise because individual results from different experiments do not agree with each other. For the mass, we obtain $1438 \pm 8$ (stat.) \pm 4 (syst.) $\mathrm{GeV}$, which can be compared with the PDG average $1425 \pm 50 \mathrm{GeV}$ and with the most precise single result, $1435 \pm 5 \mathrm{GeV}$ from the LASS Collaboration [9]. Thus, the Babar result agrees with that of LASS. For the width, we obtain $210 \pm 20$ (stat.) \pm 12 (syst.) $\mathrm{GeV}$, compared to the PDG average of $270 \pm 80 \mathrm{GeV}$ and the LASS measurement of $279 \pm 6 \mathrm{GeV}$. Thus the Babar result lies around 3 standard deviations below the LASS result.

\section{Summary}

In summary, improved results for the charged kaon electromagnetic form factor have been presented, which ex- 

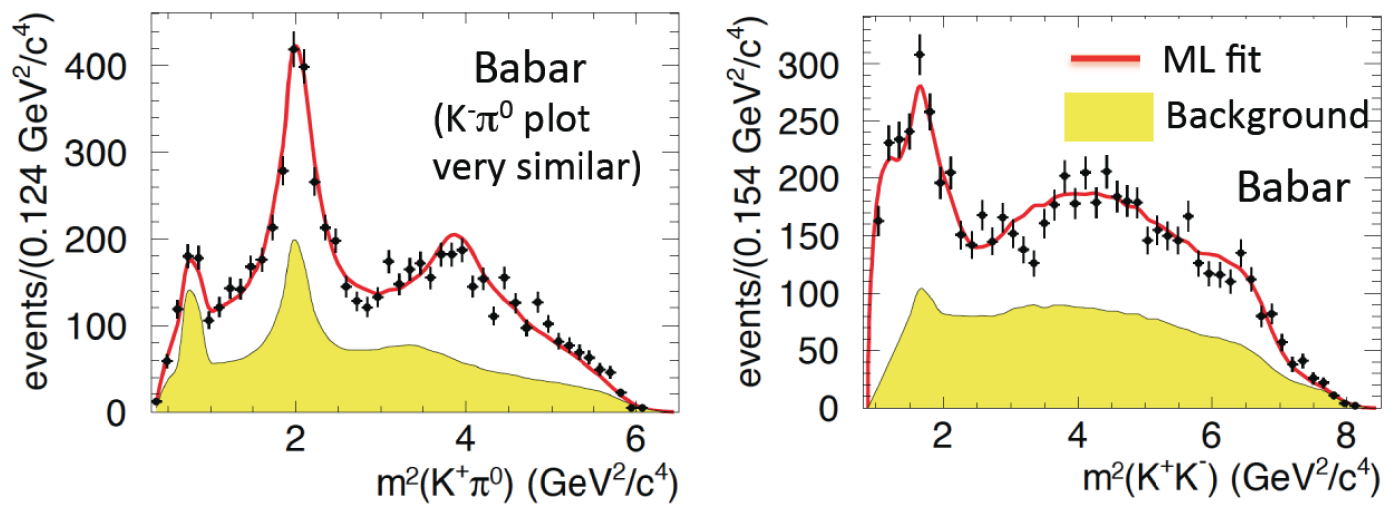

Figure 7. Projection of the Dalitz plot results for $\eta_{c} \rightarrow K^{+} K^{-} \pi^{0}$ events onto the (left) $m_{K \pi^{0}}^{2}$ and (right) $m_{K K}^{2}$ axes.

tend the measurements up to a dikaon invariant mass of $8 \mathrm{GeV}$, where the data are seen to approach the nextto-leading order QCD prediction and agree with the prediction to within the uncertainties, resolving a previous discrepancy between experiment and calculation. In addition, the first Dalitz-plot analysis of three-body $\eta_{c}$ decays has been presented. We obtain the first observation of $\eta_{c} \rightarrow K^{+} K^{-} \eta$ decays and the first evidence for $\eta_{c}(2 S) \rightarrow K^{+} K^{-} \eta$ decays. We obtain the first observation of $K_{0}^{*}(1430)^{+} \rightarrow K^{+} \eta$ decays and precise results for the $K_{0}^{*}(1430)$ mass and width. We observe the $f_{0}(1500)$ and $f_{0}(1710)$ glueball candidates in $\eta_{c}$ decays. This additional information on scalar meson production in a charmonium decays can provide new and improved results on scalar meson couplings and resonance parameters, and can help to clarify the interpretation of the scalar meson sector.

\section{References}

[1] J. P. Lees et al., (Babar Collaboration), preliminary.

[2] J. P. Lees et al., (Babar Collaboration), Phys. Rev. D 89, 112004 (2014).

[3] T. D. Pedlar et al, (CLEO Collaboration), Phys. Rev. Lett. 95, 261803 (2005).

[4] K. K. Seth et al., Phys. Rev. Lett. 110, 022002 (2013).

[5] J. P. Lees et al., (Babar Collaboration), Phys. Rev. D 88, 032013 (2013).

[6] B. Melic, B. Nizic, and K. Passek, Phys. Rev. D 60, 074004 (1999).

[7] V. L. Chernyak and A. R. Zhitnitsky, Phys. Rept. 112, 173 (1984).

[8] M. Ablikim et al. (BESIII Collaboration), Phys. Rev. D 86, 092009 (2012).

[9] D. Aston et al. (LASS Collaboration), Nucl. Phys. B 296, 493 (1988). 